\title{
A atuação do enfermeiro nos cuidados com ostomias
}

\author{
The role of nurses in ostomy care \\ El papel de las enfermeras en el cuidado de la ostomia
}

Anna Carolina Lomelino Lemos Medeiros ORCID: https://orcid.org/0000-0002-8835-9389

Universidade de Vassouras, Brasil

E-mail: carolinalomelino@outlook.com

Andréa Capistrano das Neves Cunha

ORCID: https://orcid.org/0000-0002-8396-1020

Faculdade Bezerra de Araújo, Brasil

E-mail: andcapis@hotmail.com

Angélica Cristina Castro Soares

ORCID: https://orcid.org/0000-0002-5202-6996

Universidade Castelo Branco, Brasil

E-mail: angelica.enf@outlook.com

Cristiane Alves de Sales

ORCID: https://orcid.org/0000-0001-6293-6902 Faculdade Bezerra de Araújo, Brasil

E-mail: cristiane.salles2303@gmail.com

Dayana Campos de Oliveira Cardoso ORCID: https://orcid.org/0000-0002-5031-6041 Universidade Estácio de Sá, Brasil

E-mail: camposdayana2015@ hotmail.com

Gabriela Alves Santana de Oliveira ORCID: https://orcid.org/0000-0002-9530-0924 Universidade Iguaçu, Brasil

E-mail: gabrielaalvessoliveira@gmail.com

Jacqueline Silva Araújo Lopes ORCID: https://orcid.org/0000-0003-1037-8587 Universidade Iguaçu, Brasil E-mail: jacque-music1@hotmail.com

Laís da Silva Ferreira de Souza ORCID: http://orcid.org/0000-0003-0572-7105 Universidade Iguaçu, Brasil E-mail: lais.ferreira2@hotmail.com

Larissa Machado Campana Portela ORCID: https://orcid.org/0000-0003-3248-2924 Universidade Veiga de Almeida, Brasil E-mail: larissaenfuva@hotmail.com Isabella Magalhães Barbosa

ORCID: https://orcid.org/0000-0002-7499-0890

Universidade Veiga de Almeida, Brasil E-mail: issxmagalhaes@gmail.com

Marcus Vinícius Braga Esteves ORCID: https://orcid.org/0000-0001-5450-1693 Universidade Estácio de Sá, Brasil Email:estevesbmarcus@outlook.com

Mariane Marques dos Santos Bongestad ORCID: https://orcid.org/0000-0002-7097-0497 Universidade Estácio de Sá, Brasil E-mail: gersonemariane@gmail.com

Marcelo Rodrigues Pessoa

ORCID: https://orcid.org/0000-0002-4605-5272

Universidade Estácio de Sá, Brasil

E-mail: marcelo.pesso@ hotmail.com

Raiane Monteiro Rodrigues da Silva ORCID: https://orcid.org/0000-0002-1717-7254 Universidade Iguaçu, Brasil E-mail: raiimonteiroo@gmail.com

Ronilce Rozendo Amorim ORCID: https://orcid.org/0000-0003-2291-6055

Universidade Estácio de Sá, Brasil 
Tais da Silva Araújo

ORCID: https://orcid.org/0000-0003-0303-8592 Universidade de Tecnologia e Ciências, Brasil E-mail: tais.pequenina@hotmail.com

Vítor Diego de Pontes Simões

ORCID: https://orcid.org/0000-0002-9317-4549 Centro Universitário Augusto Motta, Brasil E-mail: simoes872004@yahoo.com.br

Vitória da Silva Araujo

ORCID: https://orcid.org/0000-0003-2960-1347 Universidade Iguaçu, Brasil

E-mail: vitoriasilva178@gmail.com

Mariana Lopes Teixeira

ORCID: https://orcid.org/0000-0002-1382-3271 Universidade Estácio de Sá, Brasil E-mail: marianalopestx@gmail.com

Larissa Lessa dos Santos

ORCID: https://orcid.org/0000-0002-8947-6691 Universidade Estácio de Sá, Brasil E-mail: larissalessaa@hotmail.com

\begin{abstract}
Resumo
Introdução: Ao citar a palavra "ostomia”, entende-se por um procedimento cirúrgico realizado para exteriorizar parte de um sistema, seja respiratório, urinário ou até mesmo digestório. Estoma é a formação de uma nova abertura de forma artificial, permitindo a comunicação entre o meio interno e o externo. Objetivo: Identificar o papel do enfermeiro durante a assistência ao paciente ostomizado. Metodologia: Foi realizada uma revisão integrativa e como tática para identificação e seleção de documentos, a busca foi realizada pela busca da Biblioteca Virtual de Saúde (BVS), durante o mês de abril de 2021. Foram procurados materiais que tivessem em seu resumo os seguintes descritores em ciências da saúde (DeCS) Enfermeiro; Cuidados de Enfermagem; Ostomia. Resultados: A partir da leitura minuciosa dos artigos foram desenvolvidas duas categorias. Categoria I- As dificuldades encontradas pelo profissional enfermeiro no cuidado ao paciente ostomizado; Categoria II- A importância da enfermagem na promoção do autocuidado de indivíduos ostomizados. Considerações Finais: que a assistência de enfermagem é essencial aos indivíduos ostomizados e que mesmo com os processos técnicos fundamentados em concepções científicas efetuados pelo profissional enfermeiro, é fundamental estabelecer vínculo com seus clientes levando em conta suas questões éticas, morais, religiosas e suas personalidades singulares, tornando o cuidado individual.
\end{abstract}

Palavras-chave: Enfermeiro; Cuidados de enfermagem; Ostomia.

\begin{abstract}
Introduction: When mentioning the word "ostomy", it is understood as a surgical procedure performed to exteriorize part of a system, whether respiratory, urinary or even digestive. Stoma is the formation of a new opening artificially, allowing communication between the internal and external environment. Objective: To identify the role of nurses during the care of ostomized patients. Methodology: An integrative review was carried out and as a tactic for identification and selection of documents, the search was carried out by searching the Virtual Health Library (VHL) during the month of April 2021. Materials that had the following in their summary were sought. descriptors in health sciences (DeCS) Nurse; Nursing care; Ostomy. Results: From the thorough reading of the articles, two categories were developed. Category I- Difficulties encountered by professional nurses in caring for ostomized patients; Category II- The importance of nursing in promoting self-care for ostomized individuals. Final Considerations: that nursing care is essential for ostomized individuals and that even with the technical processes based on scientific conceptions carried out by the professional nurse, it is essential to establish a bond with their clients, taking into account their ethical, moral, religious issues and their unique personalities, making individual care.
\end{abstract}

Keywords: Nurse; Nursing care; Ostomy.

\title{
Resumen
}

Introducción: Al mencionar la palabra "ostomía", se entiende como un procedimiento quirúrgico que se realiza para exteriorizar parte de un sistema, ya sea respiratorio, urinario o incluso digestivo. El estoma es la formación de una nueva apertura de forma artificial, que permite la comunicación entre el entorno interno y externo. Objetivo: Identificar el rol del enfermero durante el cuidado de pacientes ostomizados. Metodología: Se realizó una revisión integradora y como táctica de identificación y selección de documentos se realizó la búsqueda mediante la búsqueda en la Biblioteca Virtual en Salud (BVS) durante el mes de abril de 2021. Se buscaron materiales que tuvieran lo siguiente en su resumen .descriptores en ciencias de la salud (DeCS) Enfermera; Cuidado de enfermera; Ostomía. Resultados: A partir de la lectura exhaustiva de los artículos se desarrollaron dos categorías. Categoría I- Dificultades encontradas por enfermeras profesionales en el cuidado de pacientes ostomizados; Categoría II- La importancia de la enfermería en la promoción del autocuidado de los ostomizados. Consideraciones finales: que el cuidado de 
enfermería es fundamental para los ostomizados y que aun con los procesos técnicos basados en concepciones científicas que lleva a cabo el profesional de enfermería, es fundamental establecer un vínculo con sus clientes, teniendo en cuenta sus cuestiones éticas, morales, religiosas. y sus personalidades únicas, haciendo que el cuidado sea individual.

Palabras clave: Enfermera; Cuidado de enfermera; Ostomia.

\section{Introdução}

Ao citar a palavra "ostomia", entende-se por um procedimento cirúrgico realizado para exteriorizar parte de um sistema, seja respiratório, urinário ou até mesmo digestório. Estoma é a formação de uma nova abertura de forma artificial, permitindo a comunicação entre o meio interno e o externo. (Coelho, Santos, \& Poggetto, 2013)

O enfermeiro desempenha um papel fundamental no momento do cuidado aos pacientes, pois é preciso orientá-lo sobre o autocuidado, uso de coletores e higiene, o direito de cadastramento no SUS de pessoas com estoma; pré-estabelecer referência e contrareferência caso precise de assistência. (Brasil, 2009)

Independentemente de ser definitiva ou temporária, a alteração da imagem é bem acentuada nesse tipo de procedimento, podendo gerar estado de saúde depressivo devido ao cliente sentir-se diferente do padrão da sociedade. Sabendo disso, o equilíbrio emocional deve ser trabalhando, quando possível, antes de realizar a cirurgia. (Cascais, Martini, \& Almeida, 2007)

O tratamento continuado desse paciente deve ser estimulado para que seja possível acompanhar as necessidades do indivíduo relacionadas a ostomia, pele periestomia, prevenção de complicações, apoio psicológico e até mesmo reinserção na sociedade. É de grande valia a criação de grupos de apoio para ajudá-lo a enfrentar essa nova fase. (Barbutti et al., 2008)

O profissional precisa munir o usuário de informações, orientando-o sobre sua nova condição de vida, incentivando o mesmo a realizar suas tarefas e viver a vida, mesmo com algumas adaptações. É preciso quebrar tabus e estigmas que a sociedade coloca em questão nesses casos com intuito da evolução do mesmo seja a melhor possível. (Gemelli \& Zago, 2002)

O estudo tem como objetivo identificar o papel do enfermeiro durante a assistência ao paciente ostomizado. O estudo se justifica devido ao número reduzido de pesquisas por profissionais de enfermagem na área de estomaterapia. A partir do exposto fica clara a necessidade da elaboração de trabalhos destacando a temática.

A relevância para o público acadêmico se faz com o objetivo de informar e propor uma reflexão sobre o assunto, uma vez que será de grande valia esse conhecimento para a assistência; social por levar à sociedade o conhecimento científico ao qual, de costume, ela não teria acesso e para fins de pesquisa, já que outros poderão então fazer uso das informações obtidas.

\section{Metodologia}

Bardin (2016) relata que a pesquisa qualitativa é aquela que tem fundamento principalmente nas análises qualitativas, caracterizando-a pela não utilização de instrumental estatístico na fase de análise dos dados.

Não é apenas a "pesquisa não quantitativa", tendo desenvolvido sua própria identidade. Assim, visa entender, descrever e explicar os fenômenos sociais de modos diferentes, através da análise de experiências individuais e grupais, exame de interações e comunicações que estejam se desenvolvendo, assim como da investigação de documentos (textos, imagens, filmes ou músicas) ou traços semelhantes de experiências e integrações (FLICK, 2009).

Também possui caráter descritivo, cujo foco não consiste na abordagem, mas sim no processo e seu significado, ou seja, o principal objetivo é a interpretação do fenômeno objeto de estudo (SILVA; MENEZES, 2005).

O projeto de revisão integrativa visa fazer uma interseção de estudos da mesma linha de conhecimento, com o objetivo de analisar e sintetizar os mesmos, para que se obtenha uma informação mais abrangente de um fenômeno específico, (Cooper,1984). 
A revisão integrativa sugere utilizar critérios bem definidos para as fases de coleta de dados, análise; apresentação dos resultados, desde o início da pesquisa, a partir de um protocolo de estudo previamente elaborado e validado (Ganong, 1987).

Para a elaboração deste trabalho abordou-se a proposta de revisão integrativa, que consiste na elaboração de análise abrangente de artigos, obedecendo os critérios de investigação técnico-científico para a coleta de dados, análise e apresentação dos resultados. Os dados obtidos podem sinalizar achados que precisam de mais atenção no meio acadêmico, visando melhorias da prática profissional (Ganong, 1987).

De acordo com Mendes, Silveira e Galvão (2008) a revisão de literatura deverá conter seis etapas: 1- Estabelecimento de hipótese ou questão de pesquisa; 2- Amostragem ou busca na literatura; 3- Categorização dos estudos; 4- Avaliação dos estudos incluídos na revisão; 5- Interpretação dos resultados; 6- Síntese do conhecimento ou apresentação da revisão.

Como tática para identificação e seleção de documentos, a busca foi realizada pela busca da Biblioteca Virtual de Saúde (BVS), durante o mês de abril de 2021. Foram procurados materiais que tivessem em seu resumo os seguintes descritores em ciências da saúde (DeCS) Enfermeiro; Cuidados de Enfermagem; Ostomia. Foi utilizado o boleador "and" e os critérios para seleção dos artigos foram: arquivos completos, no idioma português, com formato de artigo e recorte temporal de cinco anos (2016-2021). Os critérios de exclusão foram as pesquisas duplicadas, desatualizadas e que apresentassem divergências com o viés pesquisado neste trabalho.

Destacaram-se 8 artigos com os critérios citados anteriormente, os mesmos procederam-se à leitura detalhada para a construção do exposto, destacando os que responderam ao objetivo tendo como intuito a organização dos dados.

Os documentos foram separados por título, autores, base de dados, ano de publicação, objetivo, método de pesquisa e conclusão. A partir desse momento foi iniciada a análise bibliomética sob forma de categoria através da similaridade de cada um.

\section{Resultados e Discussão}

Os resultados apresentados no estudo foram descritos no Quadro 1.

Quadro 1: Características dos artigos analisados no período de 2010 a 2020.

\begin{tabular}{|c|c|c|c|c|c|c|}
\hline Título & Autoria & $\begin{array}{l}\text { Base de } \\
\text { dados / } \\
\text { Revista }\end{array}$ & Ano & Objetivo & Métodos & $\begin{array}{c}\text { Considerações } \\
\text { Finais }\end{array}$ \\
\hline $\begin{array}{l}\text { Aplicabilidade } \\
\text { da teoria de } \\
\text { Orem } \quad \text { no } \\
\text { autocuidado de } \\
\text { pessoa com } \\
\text { estomia } \\
\text { intestinal: } \\
\text { estudo reflexivo }\end{array}$ & $\begin{array}{l}\text { Marina } \text { Bradesco; } \\
\text { Geruza Maria da } \\
\text { Silva Gonçalves } \\
\text { Manfredini; } \\
\text { Raul de Paiva } \\
\text { Santos; } \\
\text { Zélia } \\
\text { Rodrigues } \\
\text { Silvana Resck; } \\
\text { Coelho Leite Fava; } \\
\text { Eliza Maria } \\
\text { Rezende Dázio }\end{array}$ & $\begin{array}{l}\text { Cultura de } \\
\text { los } \\
\text { Cuidados }\end{array}$ & 2020 & $\begin{array}{lr}\text { Refletir sobre } & \text { a } \\
\text { aplicabilidade } & \text { da } \\
\text { teoria do déficit } & \text { do } \\
\text { autocuidado } & \text { de } \\
\text { Dorothea Orem } & \text { no } \\
\text { cuidado } & \text { de } \\
\text { enfermagem à pessoa } \\
\begin{array}{ll}\text { com estomia } \\
\text { intestinal. }\end{array}\end{array}$ & $\begin{array}{l}\text { Revisão } \\
\text { Narrativa. }\end{array}$ & $\begin{array}{l}\text { Foi constatada a } \\
\text { potencialidade da } \\
\text { Teoria de Déficit } \\
\text { de Autocuidado } \\
\text { no planejamento } \\
\text { de ações e } \\
\text { reinserção social } \\
\text { do paciente. }\end{array}$ \\
\hline $\begin{array}{l}\text { Conhecimento } \\
\text { sobre o manejo } \\
\text { de estomias } \\
\text { intestinais de } \\
\text { eliminação }\end{array}$ & $\begin{array}{l}\text { Ana Carolina } \\
\text { Marques Oliveira; } \\
\text { Flávia Lacerda de } \\
\text { Sousa Barros; } \\
\text { Antonio Werbert } \\
\text { Silva da Costa; } \\
\text { Amanda Pereira de } \\
\text { Azevedo; } \\
\text { Paula Geórgia } \\
\text { Pereira Coelho; }\end{array}$ & $\begin{array}{l}\text { Revista de } \\
\text { Enfermage } \\
\text { UFPE On } \\
\text { Line }\end{array}$ & 2019 & $\begin{array}{lr}\text { Verificar } & \text { o } \\
\text { conhecimento } & \text { do } \\
\text { profissional de } & \text { de } \\
\text { enfermagem sobre } & \text { o } \\
\text { cuidado com pacientes } \\
\text { com estomias. }\end{array}$ & $\begin{array}{l}\text { Estudo } \\
\text { quantitativo, } \\
\text { descritivo e } \\
\text { exploratório. }\end{array}$ & $\begin{array}{lr}\text { Verificou-se } & \text { o } \\
\text { nível do } & \\
\text { conhecimento dos } \\
\text { profissionais, } \\
\text { apontandor as } \\
\text { necessidades para } \\
\text { promover } \\
\text { capacitações } \\
\text { pertinentes. }\end{array}$ \\
\hline
\end{tabular}




\begin{tabular}{|c|c|c|c|c|c|c|}
\hline & $\begin{array}{lr}\text { Monica Leal dos } \\
\text { Santos Cunha; } \\
\text { Maria } & \\
\text { Valentim } & \text { deane } \\
\text { Santos; } & \\
\text { Sarah } & \text { Nilkece } \\
\text { Mesquita } & \text { Araújo } \\
\text { Nogueira Bastos } & \\
\end{array}$ & & & & & \\
\hline $\begin{array}{lr}\text { Perfil } & \text { dos } \\
\text { usuários } & \mathrm{e} \\
\text { motivos } & \mathrm{da} \\
\text { consulta } & \mathrm{de} \\
\text { enfermagem em } & \\
\text { estomaterapia }\end{array}$ & $\begin{array}{l}\text { Rosaura } \\
\text { Paczek; } \\
\text { Andreia } \\
\text { Engelmann; } \\
\text { Giulia } \\
\text { Perini; } \\
\text { Glória Pinto } \\
\text { de Aguiar; } \\
\text { Ericarares } \\
\text { Mallmann Duarte } \\
\end{array}$ & $\begin{array}{l}\text { Revista de } \\
\text { Enfermage } \\
\text { UFPE On } \\
\text { Line }\end{array}$ & 2020 & $\begin{array}{lr}\text { Analisar o perfil } & \text { de } \\
\text { usuários e motivos } & \text { da } \\
\text { consulta } & \text { de } \\
\text { enfermagem } & \text { em } \\
\text { estomaterapia. } & \end{array}$ & $\begin{array}{l}\text { Estudo } \\
\text { quantitativo, } \\
\text { descritivo, } \\
\text { exploratório e } \\
\text { transversal. }\end{array}$ & 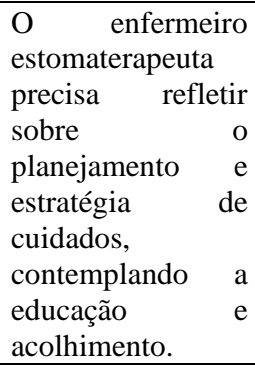 \\
\hline $\begin{array}{l}\text { Estudo quase- } \\
\text { experimental } \\
\text { com } \\
\text { enfermeiros } \\
\text { sobre estomias } \\
\text { intestinais de } \\
\text { eliminação }\end{array}$ & $\begin{array}{l}\text { Delma de Carvalho } \\
\text { Alencar }\end{array}$ & $\begin{array}{l}\text { Revista de } \\
\text { Enfermage } \\
\text { UFPE On } \\
\text { Line }\end{array}$ & 2018 & $\begin{array}{lr}\text { Avaliar o efeito da } \\
\text { educação a distância } \\
\text { no conhecimento dos } \\
\text { enfermeiros } & \text { de } \\
\text { Atenção } & \text { Primária } \\
\text { sobre } & \text { estomias } \\
\text { intestinais } & \text { de } \\
\text { eliminação. } & \end{array}$ & $\begin{array}{l}\text { Estudo quase- } \\
\text { experimental } \\
\text { do tipo antes- } \\
\text { depois. }\end{array}$ & \begin{tabular}{lr} 
Aumentar & o \\
conhecimento & dos \\
\multicolumn{2}{l}{ enfermeiros após a } \\
participação & de \\
intervenções & \\
educativas & a \\
distância & sobre \\
estomias & \\
$\begin{array}{l}\text { intestinais } \\
\text { eliminação, }\end{array}$ & de \\
melhorando & a \\
qualidade & dos \\
cuidados & \\
ofertados.
\end{tabular} \\
\hline $\begin{array}{l}\text { Complicações } \\
\text { de estomia } \\
\text { intestinal e pele } \\
\text { periestoma: } \\
\text { evidências para } \\
\text { o cuidado de } \\
\text { enfermagem }\end{array}$ & $\begin{array}{l}\text { Marina Bavaresco; } \\
\text { Geruza Maria da } \\
\text { Silva Gonçalves } \\
\text { Manfredini; } \\
\text { Camila Mendonça } \\
\text { de Moraes; } \\
\text { Rogério Silva Lima; } \\
\text { Silvana Maria } \\
\text { Coelho Leite Fava; } \\
\text { Eliza Maria } \\
\text { Rezende Dázio }\end{array}$ & $\begin{array}{l}\text { Revista de } \\
\text { Enfermagem } \\
\text { UERJ }\end{array}$ & 2019 & $\begin{array}{l}\text { Identificar e analisar } \\
\text { as evidências } \\
\text { disponíveis } \\
\text { literatura sobre as } \\
\text { complicações a de } \\
\text { estomia intestinal e } \\
\text { pele periestoma. }\end{array}$ & $\begin{array}{l}\text { Revisão } \\
\text { integrativa. }\end{array}$ & $\begin{array}{lr}\text { Notou-se que } & \text { as } \\
\text { estratégias } & \\
\text { registradas } & \text { são } \\
\text { importantes } & \text { para } \\
\text { enriquecer } & \text { as } \\
\text { consultas } & \mathrm{e} \\
\text { programas } & \\
\text { educativas. } & \end{array}$ \\
\hline \begin{tabular}{ll}
\multicolumn{2}{l}{ Aplicabilidade } \\
da Teoria de \\
Callista Roy no \\
cuidado & de \\
enfermagem & ao \\
ostomizado &
\end{tabular} & $\begin{array}{l}\text { Ana Karine da } \\
\text { Costa Monteiro; } \\
\text { Cecília Passos Vaz } \\
\text { da Costa; } \\
\text { Moniki de Oliveira } \\
\text { Barbosa Campos; } \\
\text { Ana Karoline da } \\
\text { Costa Monteiro }\end{array}$ & $\begin{array}{l}\text { Revista de } \\
\text { Enfermagem } \\
\text { e Atenção à } \\
\text { Saúde. }\end{array}$ & 2016 & $\begin{array}{lr}\text { Refletir sobre } & \text { a } \\
\text { aplicabilidade } & \text { da } \\
\text { teoria da adaptação de } \\
\text { Callista Roy } & \text { no } \\
\text { cuidado } & \text { de } \\
\text { enfermagem } & \text { ao } \\
\text { estomizado. } & \end{array}$ & $\begin{array}{l}\text { Estudo } \\
\text { teórico- } \\
\text { reflexivo. }\end{array}$ & $\begin{array}{l}\text { Acredita-se no } \\
\text { potencial da } \\
\text { aplicabilidade da } \\
\text { teoria no cuidado } \\
\text { de enfermagem ao } \\
\text { estomizado, pois } \\
\text { possibilita } \\
\text { reconhecer as } \\
\text { pessoas mediante } \\
\text { estímulos. }\end{array}$ \\
\hline $\begin{array}{l}\text { Cuidado } \\
\text { integral à } \\
\text { pessoa } \\
\text { estomizada na } \\
\text { Atenção Básica } \\
-\quad \\
\text { Conhecimento e } \\
\text { atuação do } \\
\text { enfermeiro } \\
\end{array}$ & $\begin{array}{l}\text { Lidiaine Naiara de } \\
\text { Oliveira; } \\
\text { Ana Patrícia Araújo } \\
\text { Torquato Lopes; } \\
\text { Maria das Neves } \\
\text { Decesaro }\end{array}$ & $\begin{array}{l}\text { Ciências } \\
\text { Cuidado } \\
\text { Saúde }\end{array}$ & 2017 & 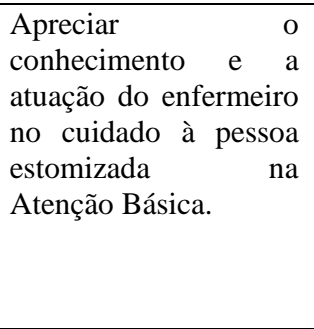 & $\begin{array}{l}\text { Pesquisa de } \\
\text { caráter } \\
\text { descritiva, } \\
\text { qualitativa e } \\
\text { exploratória. }\end{array}$ & $\begin{array}{l}\text { O estudo mostrou } \\
\text { que os } \\
\text { enfermeiros } \\
\text { possuem um } \\
\text { conhecimento } \\
\text { superficial sobre a } \\
\text { temática. }\end{array}$ \\
\hline
\end{tabular}

Fonte: Autores.

Após análise dos artigos selecionados neste estudo foram construídas as seguintes categorias: 


\section{Categoria I - As dificuldades encontradas pelo profissional enfermeiro no cuidado ao paciente ostomizado.}

Pesquisas sobre a assistência de enfermagem à paciente com ostomias apontam que existem falhas e erros no método na reabilitação do indivíduo estomizado, que podem ser determinados pelo déficit de entendimento do profissional enfermeiro sobre o tema, qualificação insatisfatória na academia ou escassez de preparo técnico e científico após se graduação. (Alencar, 2018).

O enfermeiro estomaterapeuta é referência em apoiar e reintroduzir o paciente com ostomia na coletividade, orientando para o cuidado próprio, possibilitando uma condição de vida melhor, visto que, em razão das modificações experimentadas com sua atual situação, como mudanças de sua aparência física, transformação na maneira de eliminar produtos vesicais e intestinais, há dificuldade na assistência ofertada pelos trabalhadores de saúde. (Paczek, 2020).

A assistência e orientação adequada à pessoa com estomia são fundamentais para que elas tenham confiança em seu cuidado. Além do mais, acompanhar o paciente é essencial, tanto para a instrução a respeito ao reconhecimento precoce de problemas no corpo periestoma, quanto para a sua reabilitação correta. (Bavaresco, 2019).

Destaca-se, em alguns estudos, que o enfermeiro, em atividades de estomaterapia, comumente não está ligado apenas ao cuidado direto, mas também a tarefas administrativas de planejamento da necessidade do suporte, a atribuições gerenciais e na coordenação dos materiais. (Paczek, 2020).

Alguns profissionais de enfermagem remetem-se à ostomia como sendo estritamente um orifício no intestino que se inteira com o espaço exterior e tem como finalidade a expulsão de fezes. Outros a apontam como a porta de um órgão para a parte externa do corpo. Essa análise não está totalmente incorreta, porém os trabalhadores não possuem o entendimento básico primordial para a assistência. (Oliveira, Lopes e Decesaro, 2017).

Frente à indicação quanto à orientação do indivíduo com ostomia, pesquisas exibem a inserção de serviços educativos, como o conteúdo de recuperação aprimorada no pós-cirúrgico, um avanço perioperatório categorizado com instrução longa em relação à ostomia, envolvem diminuição no período de internação e redução da quantidade de complicações referentes à ostomia. (Bavaresco, 2019).

Nota-se que existe deficiência de multidisciplinaridade e troca de saberes profissional. A atividade em conjunto viabiliza um contato maior entre enfermeiros e demais profissionais de saúde, e suas práticas criadas para cuidar do paciente com ostomia colaboram para o desenvolvimento do trabalho de saúde e para as mudanças nas intervenções que influenciam na saúde coletividade. (Oliveira, Lopes e Decesaro, 2017).

Nota-se que, além da preocupação dos trabalhadores em adquirir a aptidão na sua área, é, também, dever da empresa incentivar as pessoas na procura pela aprendizagem, oferecendo inovações contínuas para retribuir às perspectivas comercial e viabilizando uma melhora na assistência aos pacientes. (Oliveira, 2019).

Contudo, o enfermeiro se sente sem preparo para assistir pacientes com ostomia. Os motivos da reprovação e recusa aos cuidados se fundamenta pelo ensino acadêmico ineficaz, ação receptiva insuficiente e pouca contribuição em palestras, eventos e instituições. Para modificar este contexto, deve-se impulsionar a procura do estudo não só para levar o cuidado ao paciente como também apoio a educação e a pesquisa. (Monteiro et al., 2016).

\section{Categoria II - A importância da enfermagem na promoção do autocuidado de indivíduos ostomizados.}

O enfermeiro é o profissional que mais tem contato o paciente, em razão da aproximação construída no decorrer do fornecimento dos serviços. A qualidade no acolhimento de maneira programada e metódica colabora para a moldagem do indivíduo otomizado que percorre um período de transformações consequentes da elaboração da ostomia. (Monteiro et al., 2016) 
Segundo Dorothea Orem, o autocuidado se define como a execução de funções que um indivíduo efetua em pró de si mesmo, com a intenção de assegurar sua saúde, sendo esse baseado na competência para o aprendizado, nas tomadas de compreensão e decisões sobre sua nova condição. (Bavaresco et al., 2019).

$\mathrm{Na}$ fase pós-operatória, o enfermeiro resgatará a orientação do autocuidado com a ostomia e sua ação, tal como sua serventia, manuseio e substituição de materiais coletores e utilização de adjuvantes, oferecimento de indicações práticas para ajustar às circunstâncias sanitárias, recursos domiciliares destes indivíduos e imprescindibilidade de adaptações no seu cotidiano, assim como explicação de dúvida. (Alencar, 2018).

A assistência de enfermagem é competente na ascensão do bem estar e do autocuidado ao indivíduo ostomizado. O atendimento de enfermagem junto aos encontros em grupo amplia o cuidado satisfatório, proporciona cuidado à família e ampara o paciente ostomizado a dar novo significado a vida, refazer a própria imagem e resgatar a sua autoestima. (Monteiro et al., 2016).

Para o progresso de práticas que assegurem o autocuidado, é imprescindível que o profissional apoie suas condutas no saber científico, a começar das melhores comprovações e de alusivos teóricos da área, encaminhando os propósitos, as atitudes e as variadas maneiras de assistir. (Bavaresco et al., 2019).

Nas práticas educativas no ambiente ambulatorial têm de conceber lugares para a atuação do paciente e percepção sobre os seus conhecimentos e ações visto que, a conduta do profissional contribui para que o indivíduo experimente a situação de sujeito autossuficiente e independente. (Monteiro et al., 2016).

Ao prestar o cuidado, paciente e trabalhador partilham conhecimentos e estabelecem juntos quanto à aceitabilidade de determinada ação e as táticas utilizadas para a eficiência da assistência devem resultar principalmente do usuário que experimenta o cuidado. (Monteiro et al., 2016).

Assim, a oferta de qualificações através da educação continuada e permanente poderia contribuir para os enfermeiros no cuidado integral, na promoção de saúde, no domínio de patologias de base, o auxílio às complicações resultantes e a sua preparação, aperfeiçoando a particularidade de vida dos pacientes, com redução de internações por causas evitáveis e, por consequência, os consumos públicos, considerando a assistência holística aos indivíduos ostomizados. (Oliveira, Lopes e Decesaro, 2017).

\section{Conclusão}

O enfermeiro deve possuir conhecimento prático e qualificação para criar conexão e iniciar as condutas terapêuticas e construtivas com o paciente ostomizado e seus familiares. No momento inicial, seja no pós-cirúrgico ou na primeira consulta do cliente no serviço de saúde a procura de um suporte especializado a ostomizados, o diálogo deve responder o nível de compreensão que a ocasião promove.

A partir do estudo conclui-se que a assistência de enfermagem é essencial aos indivíduos ostomizados e que mesmo com os processos técnicos fundamentados em concepções científicas efetuados pelo profissional enfermeiro, é fundamental estabelecer vínculo com seus clientes levando em conta suas questões éticas, morais, religiosas e suas personalidades singulares, tornando o cuidado individual.

Mesmo com esses cuidados de enfermagem, é de grande relevância que os profissionais informem e orientem os familiares sobre o suporte e o cuidado, visto que estes se mostram importantes para a aceitação do indivíduo em uso de ostomia, obtendo autoconfiança em sua recuperação.

Entende-se que há uma grande deficiência de pesquisas para oferecer conhecimento científico aos serviços de enfermagem e que o resumo do entendimento desenvolvido até o momento será de imensa relevância para os próximos acadêmicos dessa área e contribuirá para os trabalhadores que atendem pacientes ostomizados. Recomenda-se a construção de 
Research, Society and Development, v. 10, n. 11, e600101119648, 2021

(CC BY 4.0) | ISSN 2525-3409 | DOI: http://dx.doi.org/10.33448/rsd-v10i11.19648

novos conteúdos que apresentem metodologias capazes de caracterizar fundamentações, para que dessa forma, estes sejam um auxílio seguro para considerar definições clínicas.

\section{Referências}

Bardin, L. (2016). Análise de conteúdo. Almedina Brasil.

Barbutti, S., \& Abreu. (2008). Ostomia, uma difícil adaptação. Ver. SVPH, 11(2), 27-39.

Brasil. (2009). Ministério da Saúde. Portaria No 400, de 16 de Novembro de 2009, Normatiza o atendimento à Pessoa Ostomizada no SUS. Diário Oficial da União. Brasília, DF.

Cascais, A. F., Martini, J. G., \& Almeida, P. J. (2007). O impacto da ostomia no processo de viver humano. Texto contexto enfermagem, 16 (11), $163-167$.

Coelho, A. R., Santos, F. S., \& Poggetto, M. T. (2013). A estomia mudando a vida: enfrentar para viver. REME, 17(2), 258, 267.

Cooper, H. M. (1982). Diretrizes científicas para conduzir revisões integrativas de pesquisa. Review of Educational Research. 52(2), $291-302$.

Flick. (2009). Uwe. Desenho da pesquisa qualitativa. Artmed.

Ganong, L. H. (1987). Integrative reviews of nursing research. 10(11), 1-11.

Gemelli, L. M., \& Zago, M. M. (2002). A interpretação do cuidado com o ostomizado na visão do enfermeiro: um estudo de caso. Rv. Latino-am Enfermagem, $10(1), 34-40$.

Mendes, K., Silveira, R. C., \& Galvão, C. M. (2008). Revisão integrativa: método de pesquisa para a incorporação de evidências na saúde e na enfermagem. Texto Contexto Enfermagem, 17(4): 758-64.

Silva, E. L. \& Menezes, E. M. (2005). Metodologia da pesquisa e elaboração de dissertação. (4a ed.).

Bavaresco, M. (2020). Aplicabilidade da teoria de Orem no autocuidado de pessoas com ostomia intestinal: un estudo reflexivo. Cultura dos Cuidados (Edição digital), 24 (57) http://dx.doi.org/10.14198/cuid.2020.57.21

Oliveira, A. C. (2019). Conhecimento sobre o manejo de estomias intestinais de eliminação. Rev enferm UFPE on line., 13(5):1345-53. https://doi.org/10.5205/1981-8963-v13i05a238543p1345-1353-2019

Paczek R. S., Engelmann, A. I., Perini, G. P., Aguiar, G. P. S. \& Duarte, E. R. M. (2020). Perfil de usuários e motivos da consulta de enfermagem em estomaterapia. Rev enferm UFPE on line.;14:e245710 https://doi.org/10.5205/1981-8963.2020.245710

Alencar, D. (2018). Estudo quase experimental com enfermeiros sobre estomias intestinais de eliminação. Rev enferm UFPE on line., 12(4):1191-5. https://doi.org/10.5205/1981-8963-v12i4a234972p1191-1195-2018.

Bavaresco, M. (2019). Complicações de estomia intestinal e pele periestoma: evidências para o cuidado de enfermagem. Rev enferm UERJ, $27:$ e45758. http://dx.doi.org/10.12957/reuerj.2019.45758.

Monteiro, A. K. (2016). Aplicabilidade da teoria de Callista Roy no cuidado de enfermagem ao estomizado. Rev Enferm Atenção Saúde. 5(1):84-92.

Oliveira, L. Lopes, \& A. P. Decesaro, M. (2017). Cuidado integral à pessoa estomizada na atenção básica - conhecimento e atuação do enfermeiro. Cienc Cuid Saude (3). 10.4025/cienccuidsaude.v16i3.35998. 